\title{
Synthetic Scaffolds for Pathway Enhancement
}

Ka-Hei Siu ${ }^{1}$, Rebecca P. Chen ${ }^{1}$, Qing Sun ${ }^{1}$, Long Chen ${ }^{1}$, Shen-Long Tsai ${ }^{2}$, and Wilfred Chen $^{1, *}$

1. Department of Chemical and Biomolecular Engineering, University of Delaware, Newark, DE 19716

2. Department of Chemical Engineering, National Taiwan University of Science and Technology, Taipei City, Taiwan

*Corresponding Author: wilfred@udel.edu, 302-831-6327 


\begin{abstract}
Controlling local concentrations of reactants, intermediates, and enzymes in synthetic pathways is critical for achieving satisfactory productivity of any desired products. An emerging approach to exert control over local concentrations is the use of synthetic biomolecular scaffolds to colocalize key molecules of synthetic pathways. These scaffolds bring the key molecules into close proximity by recruiting pathway enzymes via ligand binding and/or physically sequestrating enzymes and metabolites into isolated compartments. Novel scaffolds made of proteins, nucleic acids, and micro-compartments with increasingly complex architecture have recently been explored and applied to a variety of pathways, with varying degrees of success. Despite these strides, precise assembly of synthetic scaffolds remains a difficult task, particularly in vivo, where interactions both intended and unexpected can lead to unpredictable results. Additionally, because heterologous enzymes often have lowered activities in their new hosts, an ideal scaffold should provide a flexible platform that can adapt to kinetic imbalances in different contexts. In this review, we discuss some of the notable advances in the creation of these synthetic scaffolds and highlight the current challenges in their application.
\end{abstract}




\section{Introduction}

Local concentrations of reactants, intermediates, and enzymes dictate the rates and yields of all biochemical reactions. Controlling local concentrations in synthetic pathways is paramount for achieving satisfactory productivity of the desired products. Unlike natural pathways, exogenous synthetic pathways have not been adapted over eons to generate desirable products; overproduction of desirable products is rarely advantageous or necessary for survival in the host's native environments [1]. This divergence is further exacerbated as synthetic pathways become increasingly complex, introducing problems such as rapid diffusion and degradation of key intermediates, undesirable crosstalk with other cellular pathways, and accumulation of toxic metabolites that ultimately lower product yields [2]. To counter these issues, synthetic biologists have spatially organized pathway enzymes into supramolecular complexes in attempts to elevate local concentrations of enzymes and metabolites that improve reaction flux and minimize cross-reactions. To date, these efforts largely mimic natural metabolic control mechanisms that bring key components into close proximity $[3,4]$.

Analogous to naturally occurring complexes, artificial assemblies of pathway enzymes co-localize multiple molecules to enhance substrate channeling and enzyme clustering effects (See [5-7] for previous reviews). The first attempts to mimic natural complexes are simple fusions of protein domains that catalyze successive reactions. While this strategy has led to increased productivity in multiple circumstances $[8,9]$, it cannot be easily extended beyond tworeaction pathways. The strategy also precludes straightforward changes in enzyme stoichiometry, as catalytic domains are expressed in a fixed ratio on every fusion protein. Instead, synthetic biologists have taken a more modular approach using biomolecular scaffolds to co-localize target molecules [6]. Unlike simple fusions, catalytic domains are not covalently linked, but are co-localized through different interaction domains or physical sequestration. By 
decoupling binding domains and catalytic domains, generic scaffolds with multiple interaction sites can, in theory, be easily adapted to any number of synthetic pathways by simple substitution of binding and catalytic domains (Figure 1).

The potential advantages offered by this flexibility have driven the development of a wide variety of biomolecular scaffolds in recent years. Many of these scaffolds have been made from "parts" taken from interaction domains found in nature, especially protein-based scaffolds. Because the primary requirement to construct these scaffolds is proximity and interactions between the pathway enzymes, nucleic acids (DNA and RNA) $[10,11]$ and micro-compartments [12] have proven to be extremely useful as well.

Despite rapid advances in our ability to design biomolecular scaffolds a priori, particularly with nucleic acid-based scaffolds, precise assembly of synthetic pathways in vivo remains extremely challenging, primarily due to incomplete understanding of ligand-scaffold folding, unknown cross-interactions in the crowded intracellular environment, and complications resulted from adapting exogenous enzymes to host organisms. As we will discuss in this review, these challenges present both problems as well as opportunities that can be exploited using different scaffolding strategies.

\section{Protein based scaffolds}

In nature, organisms ranging from mammals to bacteria utilize enzyme complexes to optimize pathway performance [5]. The most striking examples of improved pathway efficiency by evolved enzyme complexes are those forming structures capable of physically channeling intermediates, such as carbamoyl phosphate synthase and tryptophan synthase [13]. These structures illustrate that physical tunnels can protect reactive intermediates from diffusion into the bulk solution and/or degradation from other cellular components. Another excellent example of multi-functional enzyme complexes is the natural cellulosomes of anaerobic cellulolytic 
bacteria [14]. Inspired by this natural metabolic complex, Liu et al. [15] exploited unique cohesin-dockerin interactions to assemble multi-enzyme methanol oxidation cascade containing three different $\mathrm{NAD}^{+}$-dependent dehydrogenases on the yeast cell surface. The $\mathrm{NADH}$ or electron production rate was 5 times higher than the unassembled enzyme mixture as a result of increased reaction rates facilitated by substrate channeling effects.

The most successful in vivo synthetic protein scaffolds for enhanced pathway efficiency to date are built from modular interaction domains using metazoan signaling proteins, which specifically recruit pathway enzymes tagged with their cognate interaction ligands. SH3, PDZ, and GBD domains were used by Dueber et al. [16] as interaction modules to construct synthetic protein scaffolds for mevalonate production in Escherichia coli. By optimizing synthetic scaffold expression levels to balance the scaffold to enzyme ratio and the stoichiometries of enzymes recruited to the synthetic complex, a mevalonate production $E$. coli strain was created with an astonishing 77-fold improvement in product titer [16]. The same set of scaffolds was applied inside $E$. coli cells to further improve glucaric acid productivity by about 5 -fold compared to the non-scaffolded control pathway [17]. While this enhancement demonstrates the generalizability of this set of scaffolds, these results also highlight the fact that optimal scaffold architecture for different synthetic pathways will vary. To date, optimization remains highly empirical and mechanistic understanding of scaffold function still needs more exploration [18].

Intuitively, scaffolding enhancement can be explained by proximity channeling, which involves two enzymes close enough such that the product of the former enzyme is processed by the second enzyme before it diffuses away. This mechanism has been used to explain the improvements in product titer by scaffold-mediated enzyme complexes $[15,16,17,19]$. However, proximity channeling presents a fundamental problem: an enzyme can process the diffusing substrate only within the radius $(\sim 0.1-1 \mathrm{~nm})$ of the active site. Thus, the product of the first enzyme is unlikely to be processed by the second enzyme even if their active sites are only 10 
$\mathrm{nm}$ apart [20]. As an alternative to the channeling hypothesis, Lee et al. [6] proposed a scaffoldmediated metabolite microdomain hypothesis. The central idea is that even though the intermediate produced by the former enzyme is unlikely to be processed by any individual downstream enzyme, the probability of the intermediate reacting with one of the many downstream enzymes in the micro-domain is high (Figure $2 \mathrm{~A})\left[21^{* *}\right]$. Because most enzymes naturally exist as oligomers, they can potentially form large metabolite micro-domains or enzyme agglomerates when they are bound onto multiple scaffolds [22]. For example, a PDZ domain and the corresponding PDZ ligand were used to assemble an octameric leucine dehydrogenase (LDH) with a dimeric formate dehydrogenase (FDH), providing the necessary $\mathrm{NAD}(\mathrm{H})$ recycling for the production of L-tert-leucine (Figure $2 \mathrm{~B}$ ).

Because of the uncertainty surrounding the exact mechanism to enhance pathway efficiency, current efforts to construct protein-based scaffolds for any given synthetic pathways are, at best, inexact guesswork, and difficulties are further compounded by the complexity of the crowded intracellular environment. Expanding upon the successes of protein-based scaffolds, nucleic acids-based scaffolds, which can offer a higher degree of predictability and may avoid many undesirable cross-interactions that are prevalent in protein-based scaffolds, have also been explored.

\section{Nucleic acids based scaffolds}

Use of nucleic acids as molecular scaffolds has gained a significant foothold with the advent of nucleic acid-based nanotechnology. Because DNA and RNA self-assemble in accordance to well-studied interactions, new structures can be readily designed computationally with high predictability $[23,24]$ and allows for the construction of complex nanostructures that can impose detailed spatial organization on bound proteins. 
One of the first successful demonstrations of nucleic acids as a feasible medium for pathway scaffolding involved organizing the bienzyme cascade of glucose oxidase (GOx) and horseradish peroxidase (HRP) on a long linear DNA template with two alternating docking sequences [10]. GOx and HRP were chemically modified with DNA complementary to their respective docking sites on the linear DNA template (Figure 3A). The GOx/HRP cascade was further applied to a 2-dimensional DNA scaffold composed of "hexagon-like" structures [25] (Figure 3B). Both designs enabled enzyme cascade activation while unassembled enzymes did not, a result attributed to the higher local intermediate concentrations achieved through closer enzyme proximity and further verified by another DNA origami tile scaffold study [26]. The 2D scaffolds, in particular, highlight how the rigidity of higher-dimensioned nucleic acid scaffolds provide advantages to guarantee structure and spacing over their more flexible 1-D linear counterparts.

The biggest challenge for nucleic acid scaffolds has been the adaptation of in vitro models into an in vivo context. Chemical modification techniques to link nucleic acids to enzymes in vitro can be costly and damaging to activity and are impossible to implement in vivo. Two alternative strategies for enzyme immobilization on nucleic acid scaffolds have emerged: nucleic acid binding protein domains [27*-29] and aptamer binding interactions $\left[11,30^{*}, 31\right]$. Zinc finger proteins (ZFPs), whose modular binding motifs can be engineered to recognize and bind double stranded DNA with high specificity [32], has been successfully used in conjunction with DNA-guided scaffolds to achieve in vivo synthetic pathway enhancement in E. coli $\left[27^{*}, 28\right]$. Conrado et al. used DNA scaffolds to enhance the production of three diverse metabolites: resveratrol, 1,2-propanediol, and mevalonate. By fusing ZFPs to pathway enzymes, chimeras were created and assembled onto their corresponding DNA sequences located on a plasmid DNA scaffold. The use of a plasmid DNA scaffold allowed for enzyme stoichiometry, order, and spacing to be optimized quickly and easily. 
RNA scaffolds have struggled to adopt the same strategy as DNA scaffolds due to fewer well-characterized RNA binding domains of high binding affinity. Instead, by incorporating RNA aptamers, highly specific and strong RNA binding to aptamer ligands fused to enzymes of interest can be achieved. Using this aptamer approach, RNA scaffolds have been implemented successfully in vivo to organize [FeFe]-hydrogenase and ferredoxin for hydrogen production [11]. RNA scaffolds were self-assembled in E. coli containing two unique aptamer domains that bind to their corresponding hydrogenase and ferrodoxin aptamer-binding chimeras. Discrete, one-dimensional, and two-dimensional scaffold designs enhanced hydrogen yield by 4-, 11-, and 48-fold, respectively, compared to unscaffolded enzymes, further supporting that higher ordered scaffolds provide exponentially greater enhancement.

Beyond macrostructure, the helical nature of base pairing allows for the role of enzyme orientation to be studied. The same strategy for RNA scaffolding was adopted for the production of pentadecane involving the enzymes acyl-ACP reductase (AAR) and aldehyde deformylating oxygenase (ADO) $\left[30^{* *}\right]$. The aptamer stem-loop length was modified to change the orientation of $A A R$ and $A D O$ with respect to each other. Even a one base pair difference ( $30^{\circ}$ rotation) could reduce scaffolding enhancement from 2.4 -fold to 1.5 -fold, demonstrating that nuances such as enzyme active site orientation to directly channel intermediates towards the next enzyme in the cascade rather than away can have a profound impact.

The most complex pathway implemented thus far using an in vivo nucleic acid scaffold is a four-enzyme 2-D RNA scaffold system for an $88 \%$ increase in succinate titer [30**]. While RNA based scaffolds tend to be less stable susceptible to degradation compared to DNA, transcription allows the expression of different single-stranded RNA for self-assembly. The ability to form higher-order dimensioned lattice scaffolds is crucial in achieving greater scaffolding effects., which falls in line with the scaffold-mediated metabolite microdomain hypothesis discussed in the previous section $\left[21^{\star *}\right]$. For 1D RNA scaffolds, only intermediates 
traversing on one particular "line" will encounter a downstream enzyme. Channeling intermediates down this "line" is ultimately difficult due to the flexible nature of the 1D design. In 2D and 3D RNA scaffolds, intermediates have a much higher probability of encountering their downstream enzyme partners in a manner directly proportional to the scaffold complexity because of the higher degree of enzyme clustering (Figure 3). Upcoming opportunities in this area are to design more complex architectures of higher-dimension to take advantage of macrolevel enhancements from a metabolite microdomain and to create modular templates to quickly gain understanding and optimize particular pathways of interest in terms of spacing, ordering, stoichiometry, and orientation.

\section{Micro-compartments}

While spatial organization of enzymes on protein or nucleic acid scaffolds has enhanced metabolic pathway efficiency [35], the possibility of losing intermediates/cofactors to endogenous competing pathways remains a bottleneck for further improvement in efficiency [36]. Compartmentalization provides unique advantages of physical sequestration of limiting or toxic intermediates, selective control over substrate and product fluxes in and out of the compartment, and creation of a segregated environment that is more favorable for specific reactions (Figure 4) [36,37].

Natural compartments usually consist of a porous protein shell that encapsulates enzymes. Good examples found in nature are bacterial microcompartments (MCPs) [38]. They are composed of proteins forming a shell of roughly $100-150 \mathrm{~nm}$ in diameter, with a 4-16 nm central pore on the shell that control the selective diffusion of metabolites and sequester toxic intermediates based on charge or polarity $[39,40]$. For example, the carboxysome shell forms a barrier against $\mathrm{CO}_{2}$ diffusion, which enhances carbon fixation efficiency [39]. With the discovery of targeting peptides responsible for directing native cargo proteins into MCPs $\left[41^{*}, 42\right]$, 
heterologous proteins such as green fluorescent protein and $\beta$-galactosidase have been successfully targeted and encapsulated into synthetic MCPs [43]. Additionally, engineered lumazine synthase protein capsids have been used to encapsulate HIV proteases by Wörsdörfer et al [44]. Although synthetic MCPs encapsulating a complete heterologous metabolic pathway have not yet been reported, these works serve as the framework toward more complex MCPs. Beyond enzyme manipulation, MCPs provide opportunities to control metabolite and cofactor transport across MCP shells either by customizing the residues surrounding the pores for added selectivity or by creating chimeric MCP shells with altered permeability [37].

Eukaryotes are naturally divided into intracellular compartments, and the use of mitochondria to encapsulate and cluster metabolic enzymes for enhanced pathway efficiency has been reported [45*]. The native yeast Ehrlich pathway for isobutanol production is separated into subcellular compartments: the upstream enzymes (ILV1, ILV5, ILV3) are confined to the mitochondria to generate the intermediate $\alpha$-ketoisovalerate ( $\alpha$-KIV), whereas the downstream enzymes $(\mathrm{KDC}$ and $\mathrm{ADH})$ are confined to the cytoplasm. In this configuration, a-KIV transport across the membrane becomes a major bottleneck [36]. Avalos et al. targeted $\mathrm{KDC}$ and $\mathrm{ADH}$ into the mitochondria by using the $\mathrm{N}$-terminal mitochondria localization signal sequence, thus compartmentalizing all involved enzymes and eliminating the need for intermediate transport across the mitochondrial membrane [45** $]$. Higher local enzyme and intermediate (i.e. $\alpha-K I V$ ) concentrations in the mitochondria led to a $260 \%$ enhancement in isobutaniol production compared to the native split configuration. Although similar strategies were used to implement isopentanol and 2-methyl-1-butanol pathways with $370 \%$ and $500 \%$ titer enhancement, respectively, effective use of mitochondria to host synthetic pathways is not always guaranteed, especially when substrates are not natively available or the native 
mitochondrial environment has unsuitable reaction conditions. Another potential barrier is the limited volume of most organelles relative to the whole organism [36].

In addition to modifying natural compartments, synthetic micro-compartments can be created using viral-like particles (VLPs) [35,46], polymer cages [47], or liposomes [48]. Regardless of molecular composition, all micro-compartments are intended either to encapsulate the whole pathway for higher enzyme and intermediate concentrations, or to segregate the pathway into separate environments such that each component can function at higher efficiency. One promising approach is based on the viral capsid derived from the bacteriophage $[49,50]$. High-density encapsulation of different proteins within the engineered

VLPs was demonstrated by co-expression of cargo-SP protein fusions with CP in E. coli [49]. A three-enzyme cascade catalyzing the conversion of lactose to glucose-6-phosphate was encapsulated within engineered P22 VLPs [51]. Although the overall enhancement is lower than similar scaffolding approaches, due to substantial differences in kinetic parameters among the enzymes, the P22 system provides a versatile platform for engineering synthetic MCPs for enzyme clustering.

Although ongoing research is promising, a universal platform of compartmentalization to enhance pathway efficiency remains elusive. As such, application of micro-compartments to host synthetic pathways should be designed with considerations to substrate availability, enzyme-environment interactions, transport barriers, and local concentrations of both enzymes and intermediates.

\section{Conclusions}

Synthetic pathways are, by nature, a patchwork of enzymes with markedly different reaction kinetics [52]. Although rational optimization based on modulating the absolute and relative expression levels of each enzyme has been met with some successes [53,54], the use 
of synthetic scaffolds for pathway organization has rapidly become a complementary and parallel approach to provide additional control over pathway fluxes by altering local enzyme stoichiometry. Regardless of biomolecular scaffolds employed, the central goal of exerting spatial control over enzyme clustering remains the same. While the benefit of co-localizing exogenous enzymes can be affected by many factors such as enzyme-scaffold interactions and differences in kinetic parameters, the importance of precise order and spacing of enzymes in the context of pathway enhancement remains debatable. This seeming paradox is readily observed by comparing the highly ordered nanostructures made with DNA/RNA scaffolds and the uncontrolled confinement of enzymes within micro-compartments. Before this paradox can be addressed, the experimental challenges to deconvolute multiple variables, such as relative distances, multimer interactions, and steric effects, must be met. The increasing use of nucleic acid-based scaffolds, which offer relatively predictable positional control and well-developed

analytical techniques that can track single copies of DNA/RNA molecules in vivo [55], should allow for more thorough investigations. More importantly, the possibility to assemble DNA/RNA scaffolds that are structurally tunable should pave the way for dynamic flux control of enzyme cascades in response to environmental stimuli [56].

\section{Acknowledgement}

The financial support from NSF (CBET1263774, CBET1263719, and CBET1264719) is gratefully acknowledged. 
Reference

1. Nicolaou SA, Gaida SM, Papoutsakis ET: A comparative view of metabolite and substrate stress and tolerance in microbial bioprocessing: From biofuels and chemicals, to biocatalysis and bioremediation. Metab. Eng. 2010, 12:307-331.

2. Jiang $\mathrm{H}$, Wood K V., Morgan JA: Metabolic engineering of the phenylpropanoid pathway in saccharomyces cerevisiae. Appl. Environ. Microbiol. 2005, 71:2962-2969.

3. Menon BB, Dou Z, Heinhorst S, Shively JM, Cannon GC: Halothiobacillus neapolitanus carboxysomes sequester heterologous and chimeric RubisCO species. PLoS One 2008, 3.

4. Bülow L: Characterization of an artificial bifunctional enzyme, betagalactosidase/galactokinase, prepared by gene fusion. Eur. J. Biochem. 1987, 163:443-448.

5. Conrado RJ, Varner JD, DeLisa MP: Engineering the spatial organization of metabolic enzymes: mimicking nature's synergy. Curr. Opin. Biotechnol. 2008, 19:492-499.

6. Lee H, DeLoache WC, Dueber JE: Spatial organization of enzymes for metabolic engineering. Metab. Eng. 2012, 14:242-251.

7. Na D, Kim TY, Lee SY: Construction and optimization of synthetic pathways in metabolic engineering. Curr. Opin. Microbiol. 2010, 13:363-370.

8. Meynial Salles I, Forchhammer N, Croux C, Girbal L, Soucaille P: Evolution of a Saccharomyces cerevisiae metabolic pathway in Escherichia coli. Metab. Eng. 2007, 9:152-159.

9. Wang C, Yoon S-H, Jang H-J, Chung Y-R, Kim J-Y, Choi E-S, Kim S-W: Metabolic engineering of Escherichia coli for $\alpha$-farnesene production. Metab. Eng. 2011, 13:648-655.

10. Wilner OI, Shimron S, Weizmann Y, Wang ZG, Willner I: Self-assembly of enzymes on dna scaffolds: en route to biocatalytic cascades and the synthesis of metallic nanowires. Nano Lett. 2009, 9:2040-2043. 
11. Delebecque CJ, Lindner AB, Silver PA, Aldaye FA: Organization of intracellular reactions with rationally designed RNA assemblies. Science 2011, 333:470474.

12. Parsons JB, Dinesh SD, Deery E, Leech HK, Brindley AA, Heldt D, Frank S, Smales CM, Lünsdorf H, Rambach A, et al.: Biochemical and structural insights into bacterial organelle form and biogenesis. J. Biol. Chem. 2008, 283:14366-14375.

13. Miles EW, Rhee S, Davies DR: The molecular basis of substrate channeling. J. Biol. Chem. 1999, 274:12193-12196.

14. Bayer EA, Belaich J-P, Shoham Y, Lamed R: The cellulosomes: multienzyme machines for degradation of plant cell wall polysaccharides. Annu. Rev. Microbiol. 2004, 58:521-554.

15. Liu F, Banta S, Chen W: Functional assembly of a multi-enzyme methanol oxidation cascade on a surface-displayed trifunctional scaffold for enhanced NADH production. Chem. Commun. (Camb). 2013, 49:3766-8.

16. Dueber JE, Wu GC, Malmirchegini GR, Moon TS, Petzold CJ, Ullal A V, Prather KLJ, Keasling JD: Synthetic protein scaffolds provide modular control over metabolic flux. Nat. Biotechnol. 2009, 27:753-759.

17. Moon TS, Dueber JE, Shiue E, Prather KLJ: Use of modular, synthetic scaffolds for improved production of glucaric acid in engineered $E$. coli. Metab. Eng. 2010, 12:298-305.

18. Whitaker WR, Dueber JE: Metabolic pathway flux enhancement by synthetic protein scaffolding. Methods Enzymol. 2011, 497:447-468.

19. You C, Myung S, Zhang YHP: Facilitated substrate channeling in a selfassembled trifunctional enzyme complex. Angew. Chemie - Int. Ed. 2012, 51:8787-8790.

20. Bauler P, Huber G, Leyh T, McCammon JA: Channeling by proximity: The catalytic advantages of active site colocalization using brownian dynamics. J. Phys. Chem. Lett. 2010, 1:1332-1335.

21. Castellana M, Wilson MZ, Xu Y, Joshi P, Cristea IM, Rabinowitz JD, Gitai Z, Wingreen NS: Enzyme clustering accelerates processing of intermediates through metabolic channeling. Nat. Biotechnol. 2014, 32:1011-1018. 
22. Gao X, Yang S, Zhao C, Ren Y, Wei D: Artificial Multienzyme Supramolecular Device: Highly Ordered Self-Assembly of Oligomeric Enzymes In Vitro and In Vivo. Angew. Chemie Int. Ed. 2014, 53:14027-14030.

23. Pinheiro A V., Han D, Shih WM, Yan H: Challenges and opportunities for structural DNA nanotechnology. Nat. Nanotechnol. 2011, 6:763-772.

24. Linko V, Dietz H: The enabled state of DNA nanotechnology. Curr. Opin. Biotechnol. 2013, 24:555-561.

25. Wilner OI, Weizmann Y, Gill R, Lioubashevski O, Freeman R, Willner I: Enzyme cascades activated on topologically programmed DNA scaffolds. Nat. Nanotechnol. 2009, 4:249-254.

26. Fu J, Liu M, Liu Y, Woodbury NW, Yan H: Interenzyme substrate diffusion for an enzyme cascade organized on spatially addressable DNA nanostructures. J. Am. Chem. Soc. 2012, 134:5516-5519.

27. Conrado RJ, Wu GC, Boock JT, Xu H, Chen SY, Lebar T, Turnek J, Tomšič N, Avbelj M, Gaber R, et al.: DNA-guided assembly of biosynthetic pathways promotes improved catalytic efficiency. Nucleic Acids Res. 2012, 40:18791889.

28. Lee JH, Jung SC, Bui LM, Kang KH, Song JJ, Kim SC: Improved Production of L-Threonine in Escherichia coli by Use of a DNA Scaffold System. Appl. Environ. Microbiol. 2013, 79:774-782.

29. Liu Y, Zhu Y, Ma W, Shin H dong, Li J, Liu L, Du G, Chen J: Spatial modulation of key pathway enzymes by DNA-guided scaffold system and respiration chain engineering for improved $\mathrm{N}$-acetylglucosamine production by Bacillus subtilis. Metab. Eng. 2014, 24:61-69.

30. Sachdeva G, Garg A, Godding D, Way JC, Silver P a: In vivo co-localization of enzymes on RNA scaffolds increases metabolic production in a geometrically dependent manner. Nucleic Acids Res. 2014, 42:9493-9503.

31. Delebecque CJ, Silver PA, Lindner AB: Designing and using RNA scaffolds to assemble proteins in vivo. Nat. Protoc. 2012, 7:1797-1807.

32. Negi S, Imanishi M, Matsumoto M, Sugiura $Y$ : New redesigned zinc-finger proteins: Design strategy and its application. Chem. - A Eur. J. 2008, 14:3236-3249. 
33. Feldkamp U, Niemeyer CM: Rational design of DNA nanoarchitectures. Angew. Chemie - Int. Ed. 2006, 45:1856-1876.

34. Myhrvold C, Dai M, Silver PA, Yin P: Isothermal self-assembly of complex DNA structures under diverse and biocompatible conditions. Nano Lett. 2013, 13:4242-4248.

35. Chen R, Chen Q, Kim H, Siu KH, Sun Q, Tsai SL, Chen W: Biomolecular scaffolds for enhanced signaling and catalytic efficiency. Curr. Opin. Biotechnol. 2014, 28:59-68.

36. DeLoache WC, Dueber JE: Compartmentalizing metabolic pathways in organelles. Nat. Biotechnol. 2013, 31:320-1.

37. Chen $\mathrm{AH}$, Silver PA: Designing biological compartmentalization. Trends Cell Biol. 2012, 22:662-670.

38. Kim EY, Tullman-Ercek D: Engineering nanoscale protein compartments for synthetic organelles. Curr. Opin. Biotechnol. 2013, 24:627-632.

39. Cheng S, Liu Y, Crowley CS, Yeates TO, Bobik TA: Bacterial microcompartments: Their properties and paradoxes. BioEssays 2008, 30:1084-1095.

40. Chowdhury C, Sinha S, Chun S, Yeates TO, Bobik T a.: Diverse Bacterial Microcompartment Organelles. Microbiol. Mol. Biol. Rev. 2014, 78:438-468.

41. Parsons JB, Frank S, Bhella D, Liang M, Prentice MB, Mulvihill DP, Warren MJ: Synthesis of Empty Bacterial Microcompartments, Directed Organelle Protein Incorporation, and Evidence of Filament-Associated Organelle Movement. Mol. Cell 2010, 38:305-315.

42. Fan C, Cheng S, Liu Y, Escobar CM, Crowley CS, Jefferson RE, Yeates TO, Bobik TA: Short $\mathbf{N}$-terminal sequences package proteins into bacterial microcompartments. Proc. Natl. Acad. Sci. U. S. A. 2010, 107:7509-7514.

43. Choudhary S, Quin MB, Sanders MA, Johnson ET, Schmidt-Dannert C: Engineered protein nano-compartments for targeted enzyme localization. PLoS One 2012, 7.

44. Wörsdörfer B, Woycechowsky KJ, Hilvert D: Directed evolution of a protein container. Science 2011, 331:589-592. 
45. Avalos JL, Fink GR, Stephanopoulos G: Compartmentalization of metabolic pathways in yeast mitochondria improves the production of branched-chain alcohols. Nat. Biotechnol. 2013, 31:335-41.

46. Rhee J-K, Hovlid M, Fiedler JD, Brown SD, Manzenrieder F, Kitagishi H, Nycholat C, Paulson JC, Finn MG: Colorful virus-like particles: fluorescent protein packaging by the Q $\beta$ capsid. Biomacromolecules 2011, 12:3977-81.

47. Quellec P, Gref R, Dellacherie E, Sommer F, Tran MD, Alonso MJ: Protein encapsulation within poly(ethylene glycol)-coated nanospheres. II. Controlled release properties. J. Biomed. Mater. Res. 1999, 47:388-395.

48. Colletier J-P, Chaize B, Winterhalter M, Fournier D: Protein encapsulation in liposomes: efficiency depends on interactions between protein and phospholipid bilayer. BMC Biotechnol. 2002, 2:9.

49. O'Neil A, Reichhardt C, Johnson B, Prevelige PE, Douglas T: Genetically programmed in vivo packaging of protein cargo and its controlled release from bacteriophage P22. Angew. Chemie - Int. Ed. 2011, 50:7425-7428.

50. Patterson DP, Prevelige PE, Douglas T: Nanoreactors by programmed enzyme encapsulation inside the capsid of the bacteriophage P22. ACS Nano 2012, 6:5000-5009.

51. Patterson DP, Schwarz B, Waters RS, Gedeon T, Douglas T: Encapsulation of an enzyme cascade within the bacteriophage $\mathrm{P} 22$ virus-like particle. ACS Chem. Biol. 2014, 9:359-365.

52. Moon TS, Yoon SH, Lanza AM, Roy-Mayhew JD, Jones Prather KL: Production of glucaric acid from a synthetic pathway in recombinant Escherichia coli. Appl. Environ. Microbiol. 2009, 75:589-595.

53. Pitera DJ, Paddon CJ, Newman JD, Keasling JD: Balancing a heterologous mevalonate pathway for improved isoprenoid production in Escherichia coli. Metab. Eng. 2007, 9:193-207.

54. Pfleger BF, Pitera DJ, Smolke CD, Keasling JD: Combinatorial engineering of intergenic regions in operons tunes expression of multiple genes. Nat. Biotechnol. 2006, 24:1027-1032.

55. Golding I, Cox EC: RNA dynamics in live Escherichia coli cells. Proc. Natl. Acad. Sci. U. S. A. 2004, 101:11310-11315. 


\section{Qi LS, Arkin AP: A versatile framework for microbial engineering using synthetic non-coding RNAs. Nat. Rev. Microbiol. 2014, 12:341-54.}

**21. Castellana et al., 2014:

The authors developed a quantitative model to assess the benefits of enzyme clustering and predicted that clustered enzymes increase the rate of intermediate processing and metabolic efficiency for a two-step and a three-step pathway.

*27. Conrado et al., 2012:

A plasmid DNA scaffold system was used in vivo allowed enzyme stoichiometry, order, and spacing to be easily adapted and optimized to zinc finger protein chimera sets of three diverse metabolic pathways.

**30. Sachdeva et al., 2014:

The authors assembled RNA scaffolds comprised of polymerization domains and aptamers in vivo of $E$. coli and proposed a model to illustrate the importance of the relative orientation of enzyme active sites.

*42. Fan et al., 2010:

The authors reported a short N-terminal sequence from Salmonella enterica Pdu enzyme is necessary and sufficient to direct GFP, GST, and MBP to the lumen of Pdu micro-compartment.

**45. Avalos et al., 2013:

The authors showed that targeting of the Ehrlich pathway enzymes in yeast into mitochondria increased isobutanol production by $260 \%$ compared to overexpression of the enzymes in cytoplasm, highlighting the potential benefits of compartmentalization. 
Fig. 1

A) Free enzymes

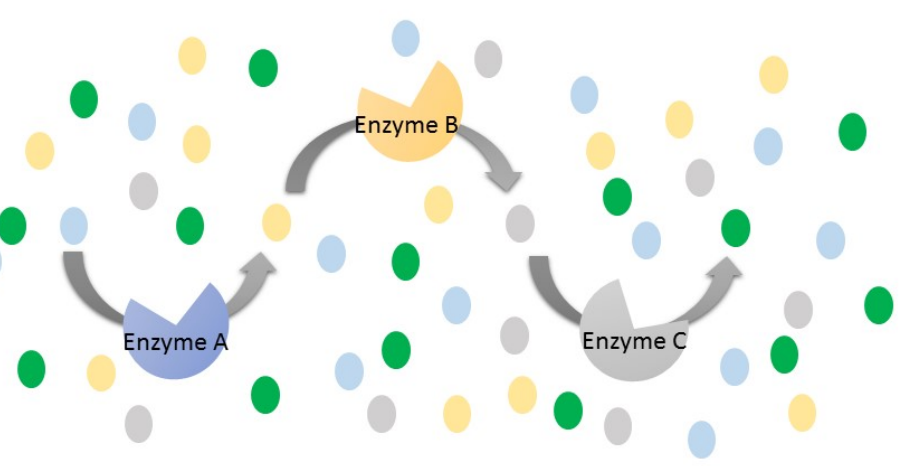

B) Direct Fusions
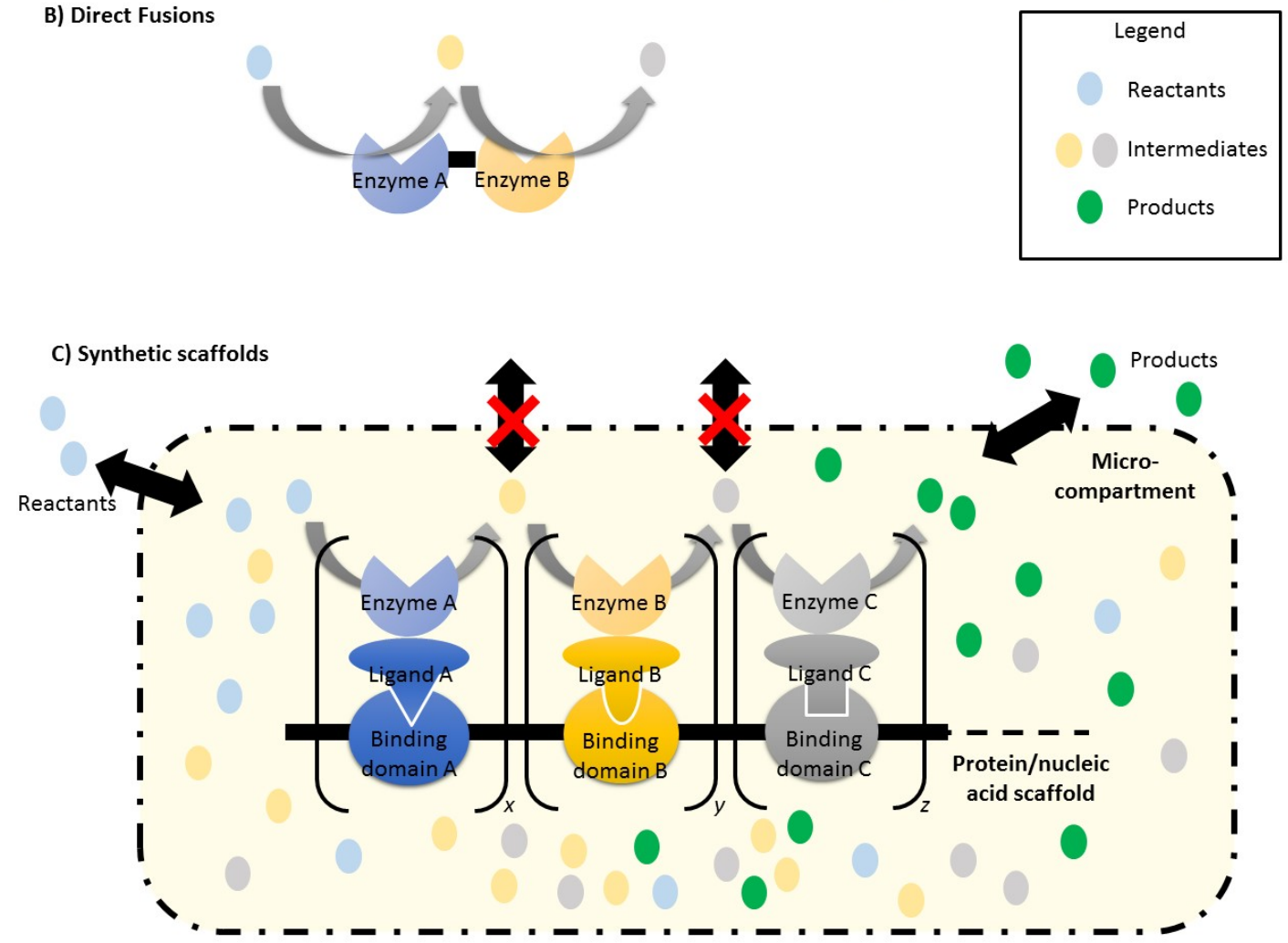

Figure 1. Spatial organizations of pathway enzymes. A) Pathway enzymes are "freely soluble" and their substrates also freely diffuse in the solution. There is no spatial control over local concentrations and, thus, reaction rates are determined by bulk solution conditions and could be reduced by factors listed in text. B) Direct fusions of enzymes provide so-called "substrate channeling" effect and limit exposure of intermediates to potential losses. However, fusions do not allow straightforward changes to enzyme stoichiometry. C) Pathway enzymes are organized onto synthetic scaffolds. Both enzymes and substrates are co-localized by non-covalent binding and/or physical sequestration. Local concentrations of key molecules are elevated to potentially improve reaction fluxes and balance kinetic parameters, in part by changing the number of enzymes $(x, y, z)$ on each scaffold assembly. 
Figure 2

A) Microdomain Hypothesis
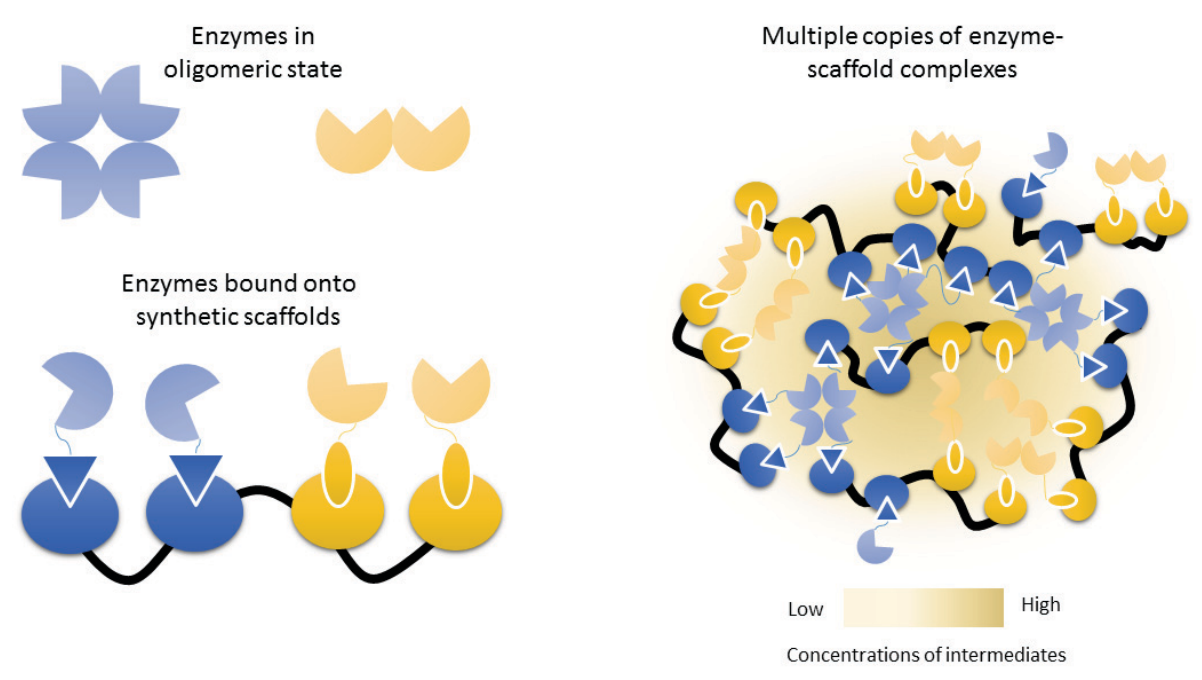

Concentrations of intermediates
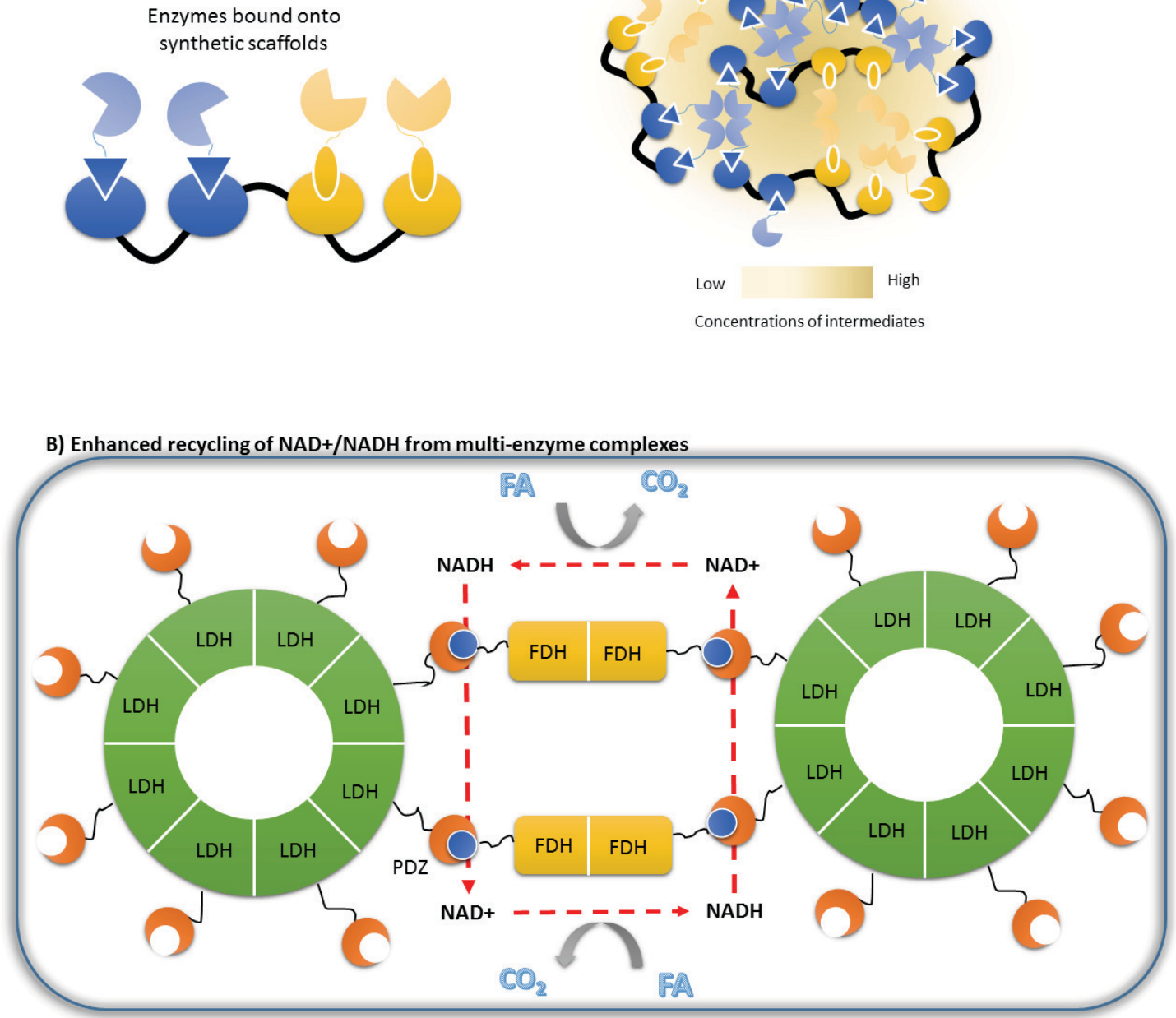

Figure 2. Enzyme-ligand fusions can form large metabolite micro-domains or enzyme agglomerates when they are bound onto multiple scaffolds. A) Because many pathway enzymes naturally exist as oligomers, multimers of enzyme-ligand fusions can link with each other to form local regions of elevated metabolite concentrations and limit loss of intermediates. Quantitative modeling by Castellana et al., 2014 [30**] suggests multi-enzyme clusters that may be formed by enzyme-scaffold complexes can achieve rapid processing of intermediates. B) In this example, an octameric LDH and a dimeric LDH self-assemble into large complexes that facilitate 2-fold higher NADH-recycling efficiency and L-tert-leucine production by restricting NAD+/NADH diffusion out of micro-domains. (Adapted from Gao et al., 2014) 
Figure 3

A) 1D scaffold

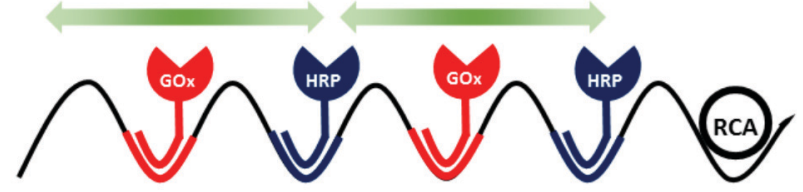

B) 2D scaffold
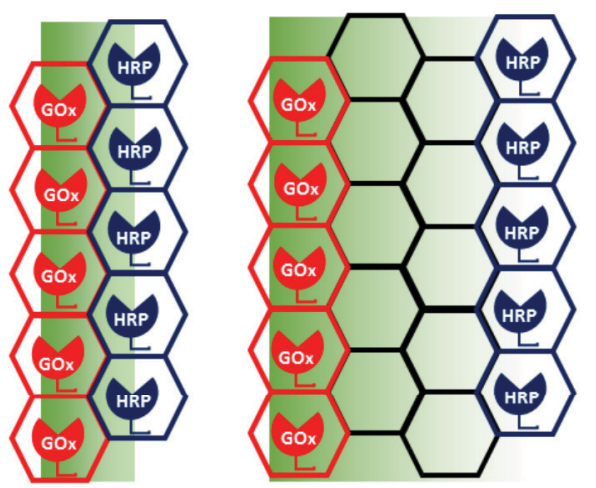

Figure 3. Multi-dimensional nucleic acid scaffolds. A) Linear one-dimensional single-stranded DNA scaffold created through rolling circle amplification (RCA). Glucose oxidase (GOx) and horseradish peroxidase (HRP) were chemically conjugated with oligonucleotides to bind onto specific sites on the linear scaffold. Substrate channeling enhancement is effective along a "line". B) Hexagonal-structured two-dimensional DNA scaffold self-assembled through strands with partial complimentarity. GOx and HRP were docked on 2-hexagonal and 4-hexagonal scaffolds, with the 2-hexangonal design providing greater enhancement because the closer proximity of HRP encounters a higher effective concentration of the intermediate. Substrate channeling is effective along a "plane", thus providing more enhancement over linear scaffolds. 


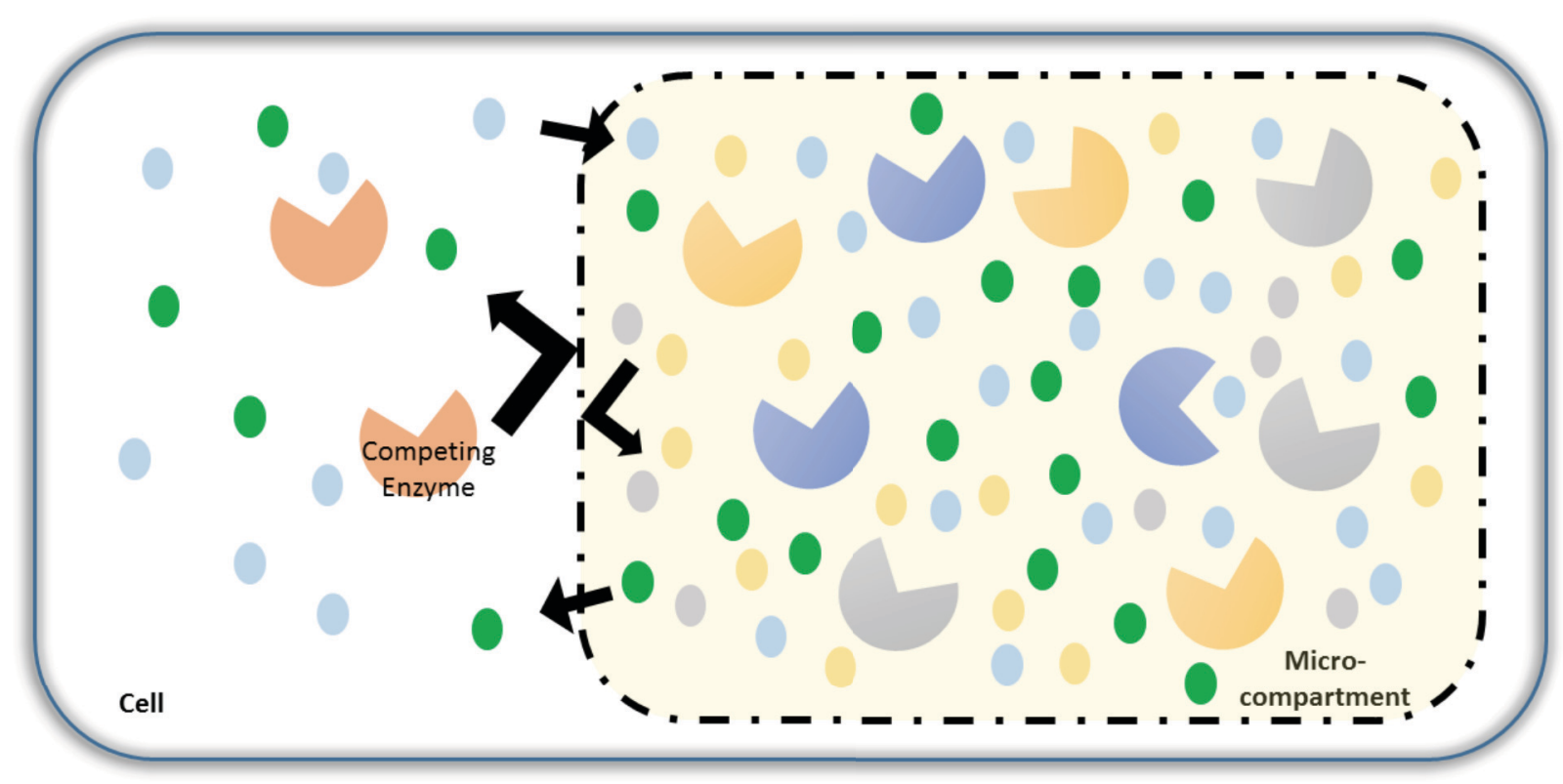

Figure 4. Micro-compartment confines pathway enzymes and intermediates into a physically isolated environment separate from other cell components. The isolated environment can be made to promote activities of the pathway enzymes within the compartment. This physical sequestration also protect enzyme and intermediate from losses due to competing reactions and degradation as well as elevating local concentrations of key molecules. As such, fluxes across the selective molecular barrier plays a significant role in the effectiveness of these scaffolds. (Adapted from Chen A.H. et al., 2012) 


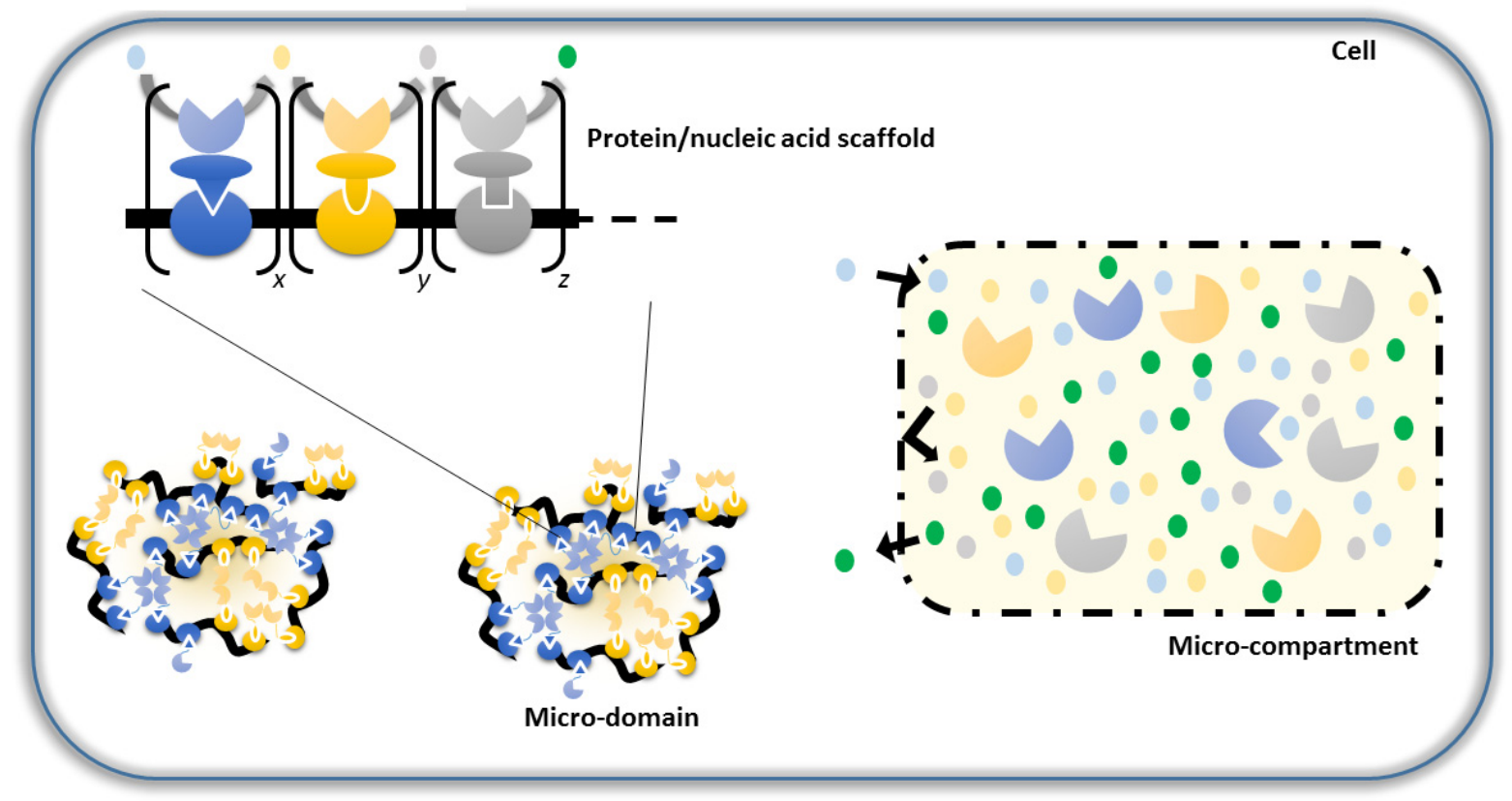

